\title{
Local solvability and stability of the inverse problem for the non-self-adjoint Sturm-Liouville operator
}

Natalia P. Bondarenko ${ }^{1,2^{*}}$ (D)

*Correspondence:

BondarenkoNP@info.sgu.ru

'Department of Applied

Mathematics and Physics, Samara

National Research University,

Moskovskoye Shosse 34, Samara

443086, Russia

${ }^{2}$ Department of Mechanics and

Mathematics, Saratov State

University, Astrakhanskaya 83,

Saratov 410012, Russia

\begin{abstract}
We consider the non-self-adjoint Sturm-Liouville operator on a finite interval. The inverse spectral problem is studied, which consists in recovering this operator from its eigenvalues and generalized weight numbers. We prove local solvability and stability of this inverse problem, relying on the method of spectral mappings. Possible splitting of multiple eigenvalues is taken into account.
\end{abstract}

MSC: 34A55; 34B09; 34B24; 34L40

Keywords: Inverse spectral problems; Non-self-adjoint Sturm-Liouville operator; Generalized spectral data; Local solvability; Stability; Method of spectral mappings; Boundary value problem

\section{Introduction}

The paper concerns the theory of inverse spectral problems for differential operators. Such problems consist in constructing operators by their spectral information.

We consider the Sturm-Liouville boundary value problem (BVP) $L=L(q(x), h, H)$ endowed with Robin boundary conditions (see, e.g., [1]) in the following form:

$$
\begin{aligned}
& -y^{\prime \prime}+q(x) y=\lambda y, \quad x \in(0, \pi), \\
& y^{\prime}(0)-h y(0)=0, \quad y^{\prime}(\pi)+H y(\pi)=0 .
\end{aligned}
$$

Here, $\lambda$ is the spectral parameter, $q$ is the complex-valued function from $L_{2}(0, \pi)$, called the potential, $h$ and $H$ are complex numbers. Denote by $\left\{\lambda_{n}\right\}_{n=0}^{\infty}$ the eigenvalues of $L$ counted with their multiplicities and numbered so that $\left|\lambda_{n}\right| \leq\left|\lambda_{n+1}\right|, n \geq 0$. The eigenvalue problem (1.1)-(1.2) appears after separation of variables in problems of mathematical physics, describing wave propagation, heating, and other processes. More information about applications and generalizations of the Sturm-Liouville boundary value problems can be found, e.g., in the recent papers [2-4].

The most complete results on inverse problems of spectral analysis have been obtained for self-adjoint Sturm-Liouville operators. Those results include uniqueness theorems,

(c) The Author(s) 2020. This article is licensed under a Creative Commons Attribution 4.0 International License, which permits use, sharing, adaptation, distribution and reproduction in any medium or format, as long as you give appropriate credit to the original author(s) and the source, provide a link to the Creative Commons licence, and indicate if changes were made. The images or other third party material in this article are included in the article's Creative Commons licence, unless indicated otherwise in a credit line to the material. If material is not included in the article's Creative Commons licence and your intended use is not permitted by statutory regulation or exceeds the permitted use, you will need to obtain permission directly from the copyright holder. To view a copy of this licence, visit http://creativecommons.org/licenses/by/4.0/. 
algorithms for constructive solution, spectral data characterization, local solvability and stability (see studies [5-11] and the references therein).

Let us formulate one of the classical inverse problems for $L$ in the self-adjoint case (i.e., when $q(x)$ for $x \in(0, \pi), h$ and $H$ are real). In this case, all the eigenvalues $\left\{\lambda_{n}\right\}_{n=0}^{\infty}$ are real and simple. Let $\varphi(x, \lambda)$ be the solution of equation (1.1), satisfying the initial conditions $\varphi(0, \lambda)=1, \varphi^{\prime}(0, \lambda)=h$. Define the weight numbers

$$
\alpha_{n}:=\int_{0}^{\pi} \varphi^{2}\left(x, \lambda_{n}\right) d x, \quad n \geq 0
$$

Inverse problem 1.1 Given the data $\left\{\lambda_{n}, \alpha_{n}\right\}_{n=0}^{\infty}$, construct $q(x), h$, and $H$.

Inverse problem 1.1 is equivalent to the inverse problem by the spectral function, studied by Marchenko [5].

In the non-self-adjoint case, some of the eigenvalues $\left\{\lambda_{n}\right\}_{n=0}^{\infty}$ can be multiple, so the problem becomes more difficult to investigate. There are significantly less studies on inverse problems for the non-self-adjoint operator (1.1)-(1.2). In particular, in [8] the classical results for the self-adjoint Sturm-Liouville operator are generalized to the non-self-adjoint case with simple eigenvalues. Tkachenko [12] developed the method for solving another inverse problem, which consists in recovering the non-self-adjoint Sturm-Liouville operator from two spectra corresponding to different boundary conditions.

Note that the spectral data $\left\{\lambda_{n}, \alpha_{n}\right\}_{n=0}^{\infty}$ defined above do not uniquely specify $q, h$, and $H$ in the general case. Nevertheless, in [13] the so-called generalized spectral data (GSD) has been introduced in the following way. Without loss of generality, we assume that multiple eigenvalues are consecutive: $\lambda_{n}=\lambda_{n+1}=\cdots=\lambda_{n+m_{n}-1}$, where $m_{n}$ is the multiplicity of the eigenvalue $\lambda_{n}$. By virtue of the well-known asymptotics

$$
\sqrt{\lambda_{n}}=n+O\left(n^{-1}\right), \quad n \rightarrow \infty
$$

we have $m_{n}=1$ for sufficiently large $n$. Define

$$
\begin{aligned}
& S:=\{0\} \cup\left\{n \in \mathbb{N}: \lambda_{n} \neq \lambda_{n-1}\right\}, \\
& \varphi_{n+\nu}(x):=\frac{1}{\nu !} \frac{d^{\nu}}{d \lambda^{\nu}} \varphi(x, \lambda)_{\mid \lambda=\lambda_{n}}, \quad n \in S, v=\overline{0, m_{n}-1} .
\end{aligned}
$$

The sequence $\left\{\varphi_{n}\right\}_{n=0}^{\infty}$ is a complete system of root-functions for the problem $L$. The generalized weight numbers are defined as follows:

$$
\alpha_{n+v}=\int_{0}^{\pi} \varphi_{n+v}(x) \varphi_{n+m_{n}-1}(x) d x, \quad n \in S, v=\overline{0, m_{n}-1} .
$$

Clearly, definition (1.5) generalizes (1.3).

Thus, Inverse problem 1.1 turns into the inverse problem by GSD $\left\{\lambda_{n}, \alpha_{n}\right\}_{n=0}^{\infty}$. Buterin [13] has proved the uniqueness theorem for this inverse problem and obtained a constructive algorithm for its solution based on the method of spectral mappings [8, 14]. The question of GSD characterization for Sturm-Liouville operators with complex-valued potentials was investigated in $[15,16]$. However, necessary and sufficient conditions on GSD 
from $[15,16]$ require solvability of some main equations. Those requirements are difficult to verify.

The aim of this paper is to investigate local solvability and stability of Inverse problem 1.1 in the non-self-adjoint case. Note that, under a small perturbation of the spectrum, multiple eigenvalues can split into smaller groups, so the generalized weight numbers change their form. As far as we know, this effect has not been studied before.

Some fragmentary results on stability under splitting of multiple eigenvalues were obtained in [17-19] for various inverse problems. Recently Buterin and Kuznetsova [20] proved local solvability and stability for the inverse problem by two spectra for the nonself-adjoint Sturm-Liouville operator. They also took splitting of multiple eigenvalues into account. However, Inverse problem 1.1 appears to be more interesting for investigation because of generalized weight numbers changing their structure.

In [15], some results on local solvability and stability were obtained for the inverse problem of recovering the non-self-adjoint Sturm-Liouville operator with the Dirichlet boundary conditions from GSD. However, the authors of [15] considered only such perturbations of GSD that preserve eigenvalue multiplicities. In the present paper, arbitrary perturbations that can change eigenvalue multiplicities are studied. We obtain special conditions on a GSD perturbation, which allow GSD to change their structure, but the perturbation of the potential remains small in $L_{2}$-norm. In our sequel studies [21, 22], the results of this paper are applied to investigate the non-self-adjoint Sturm-Liouville problem with arbitrary entire functions in the boundary condition.

The paper is organized as follows. In Sect. 2, our main Theorems 2.2 and 2.3 on local solvability and stability are formulated. Their proofs are constructive and develop the ideas of the method of spectral mappings $[8,13,14]$. This method consists in reduction of a nonlinear inverse problem to a linear equation in a Banach space, called the main equation. The main equation and the corresponding Banach space are specially constructed for every certain inverse problem. For our problem, the most important feature of the main equation is that it contains "continuous" and "discrete" components. Derivation of the main equation and the proof of its unique solvability are provided in Sect. 3. In Sect. 4, we finish the proofs of Theorems 2.2 and 2.3, and consider an example. In Sect. 5, analogous results are formulated for equation (1.1) with the Dirichlet boundary conditions.

\section{Main results}

We start with some preliminaries. Let $\Phi(x, \lambda)$ be the solution of Eq. (1.1) satisfying the boundary conditions $\Phi^{\prime}(0, \lambda)-h \Phi(0, \lambda)=1, \Phi^{\prime}(\pi, \lambda)+H \Phi(\pi, \lambda)=0$. The function $M(\lambda):=$ $\Phi(0, \lambda)$ is called the Weyl function of the problem $L$. Weyl functions are natural spectral characteristics for various self-adjoint and non-self-adjoint operators (see [5, 8]). It is easy to show that $\Phi(x, \lambda)$ for each fixed $x \in[0, \pi]$ and $M(\lambda)$ are meromorphic functions in the $\lambda$-plane having the poles at $\lambda=\lambda_{n}, n \geq 0$. In [13], the following representation has been obtained:

$$
M(\lambda)=\sum_{n \in S} \sum_{\nu=0}^{m_{n}-1} \frac{M_{n+\nu}}{\left(\lambda-\lambda_{n}\right)^{\nu+1}} .
$$


The coefficients $\left\{M_{n}\right\}_{n=0}^{\infty}$ can be uniquely determined by the generalized weight numbers $\left\{\alpha_{n}\right\}_{n=0}^{\infty}$ and vice versa from the linear system

$$
\sum_{k=0}^{v} \alpha_{n+v-k} M_{n+m_{n}-k-1}=\delta_{v, 0}, \quad n \in S, v=\overline{0, m_{n}-1} .
$$

In particular, $M_{n}=\alpha_{n}^{-1}$, if $m_{n}=1$. Thus, Inverse problem 1.1 by the GSD is equivalent to the following one.

Inverse problem 2.1 Given the data $G:=\left\{\lambda_{n}, M_{n}\right\}_{n=0}^{\infty}$, find $q, h$, and $H$.

Further we study Inverse problem 2.1 instead of Inverse problem 1.1.

Along with the problem $L$, we consider complex numbers $\tilde{G}=\left\{\tilde{\lambda}_{n}, \tilde{M}_{n}\right\}_{n=0}^{\infty}$. We will show that, if the data $\tilde{G}$ are "sufficiently close" to $G=\left\{\lambda_{n}, M_{n}\right\}_{n=0}^{\infty}$ in some sense (a rigorous formulation is given in Theorem 2.2), then $\tilde{G}$ will correspond to some BVP $\tilde{L}=L(\tilde{q}(x), \tilde{h}, \tilde{H})$ of the same form as $L$, but with different coefficients. We agree that, if a certain symbol $\gamma$ is related to $L$, then the symbol $\tilde{\gamma}$ with tilde is the analogous object constructed by the data $\tilde{G}$.

Let $N=N(L) \geq 0$ be the minimal integer such that $m_{n}=1$ for $n>N$ and $\left|\lambda_{N}\right|<\left|\lambda_{N+1}\right|$. Fix any real $r \in\left(\left|\lambda_{N}\right|,\left|\lambda_{N+1}\right|\right)$, and consider the contour $\gamma_{N}=\gamma_{N}(L)=\{\lambda \in \mathbb{C}:|\lambda|=r\}$ surrounding the eigenvalues $\left\{\lambda_{n}\right\}_{n=0}^{N}$. Put

$$
\begin{aligned}
& S_{N}:=S \cap\{0, \ldots, N\}, \quad \tilde{S}_{N}:=\tilde{S} \cap\{0, \ldots, N\}, \\
& \mathcal{M}_{N}(\lambda):=\sum_{n \in S_{N}} \sum_{v=0}^{m_{n}-1} \frac{M_{n+v}}{\left(\lambda-\lambda_{n}\right)^{v+1}}, \quad \tilde{\mathcal{M}}_{N}(\lambda):=\sum_{n \in \tilde{S}_{N}} \sum_{v=0}^{\tilde{m}_{n}-1} \frac{\tilde{M}_{n+v}}{\left(\lambda-\tilde{\lambda}_{n}\right)^{v+1}}, \\
& \hat{\mathcal{M}}_{N}:=\tilde{\mathcal{M}}_{N}-\mathcal{M}_{N} .
\end{aligned}
$$

Note that the function $\hat{\mathcal{M}}_{N}(\lambda)$ is constructed by the data $\left\{\lambda_{n}, M_{n}\right\}_{n=0}^{N}$ and $\left\{\tilde{\lambda}_{n}, \tilde{M}_{n}\right\}_{n=0}^{N}$. Define $\rho_{n}:=\sqrt{\lambda_{n}}$, arg $\rho_{n} \in\left[-\frac{\pi}{2}, \frac{\pi}{2}\right)$, and $\xi_{n}:=\left|\rho_{n}-\tilde{\rho}_{n}\right|+\left|M_{n}-\tilde{M}_{n}\right|$ for $n \geq 0$.

Theorem 2.2 Let $L=L(q(x), h, H)$ be a fixed BVP in the form (1.1)-(1.2), $N=N(L)$, $\gamma_{N}=\gamma_{N}(L)$. Then there exists $\delta_{0}>0$ (depending on $L$ ) such that, for any $\delta \in\left(0, \delta_{0}\right]$ and any complex numbers $\tilde{G}=\left\{\tilde{\lambda}_{n}, \tilde{M}_{n}\right\}_{n=0}^{\infty}$ satisfying the conditions

$$
\begin{aligned}
& \max _{\lambda \in \gamma_{N}}\left|\hat{\mathcal{M}}_{N}(\lambda)\right| \leq \delta, \\
& \left(\sum_{n=N+1}^{\infty}\left(n \xi_{n}\right)^{2}\right)^{1 / 2} \leq \delta,
\end{aligned}
$$

there exist a complex-valued function $\tilde{q} \in L_{2}(0, \pi)$ and complex numbers $\tilde{h}, \tilde{H}$ being the solution of Inverse problem 2.1 for $\tilde{G}$. Moreover,

$$
\|q-\tilde{q}\|_{L_{2}(0, \pi)} \leq C \delta, \quad|h-\tilde{h}| \leq C \delta, \quad|H-\tilde{H}| \leq C \delta .
$$

Here and below, the same symbol $C$ is used for various positive constants depending on $L$ and $\delta_{0}$ and independent of $\delta, \tilde{G}$, etc. 
Recall that the function $\mathcal{M}_{N}(\lambda)$ is fixed and all its poles lie inside $\gamma_{N}$. Condition (2.2) for sufficiently small $\delta$ implies that all the poles of $\tilde{\mathcal{M}}_{N}(\lambda)$ also lie inside $\gamma_{N}$. Moreover, the following estimate holds:

$$
\left|\lambda_{n}-\tilde{\lambda}_{n}\right| \leq C \delta^{1 /(N+1)}, \quad n=\overline{0, N} .
$$

However, the values $\lambda_{n}$ and $\tilde{\lambda}_{n}$ can have different multiplicities. Namely, multiple values $\lambda_{n}$ can split into smaller groups, so $S \subseteq \tilde{S}, \tilde{m}_{n}=m_{n}=1$ for all $n>N$.

We also obtain local solvability and stability conditions on the discrete data, not involving the continuous function $\hat{\mathcal{M}}_{N}$. Such conditions are provided in the following theorem.

Theorem 2.3 Let $L=L(q(x), h, H)$ be a fixed BVP in the form (1.1)-(1.2), $N=N(L)$. Then there exists $\delta_{0}>0$ (depending on $L$ ) such that, for any $\delta \in\left(0, \delta_{0}\right]$ and any complex numbers $\tilde{G}=\left\{\tilde{\lambda}_{n}, \tilde{M}_{n}\right\}_{n=0}^{\infty}$ satisfying conditions (2.3) and

$$
\left.\begin{array}{l}
\tilde{\lambda}_{n} \neq \tilde{\lambda}_{k}, \quad n \neq k, n, k \geq 0, \\
\left|\sum_{j=0}^{m_{k}-1}\left(\tilde{\lambda}_{k+j}-\lambda_{k}\right)^{s} \tilde{M}_{k+j}-M_{k+s}\right| \leq \delta, \quad s=\overline{0, m_{k}-1}, \\
\left|\sum_{j=0}^{m_{k}-1}\left(\tilde{\lambda}_{k+j}-\lambda_{k}\right)^{s} \tilde{M}_{k+j}\right| \leq \delta, \quad s=\overline{m_{k}, 2\left(m_{k}-1\right)}, \quad k \in S_{N}, \\
\left|\tilde{\lambda}_{k+j}-\lambda_{k}\right| \leq \delta^{1 / m_{k},} \quad\left|\tilde{M}_{k+j}\right| \leq \delta^{\left(1-m_{k}\right) / m_{k}},
\end{array}\right\}
$$

there exist a complex-valued function $q \in L_{2}(0, \pi)$ and complex numbers $h, H$ being the solution of Inverse problem 2.1 for $\tilde{G}$. Moreover, estimates (2.4) hold.

Condition (2.5) is imposed for simplicity. One can similarly consider the case of multiple values among $\left\{\tilde{\lambda}_{n}\right\}_{n=0}^{N}$, but then one needs more complicated requirements instead of relations (2.6).

Theorems 2.2 and 2.3 generalize their analogue for the self-adjoint case [8, Theorem 1.6.4].

\section{Main equation}

The goal of this section is to derive the main equation in a Banach space, which plays a crucial role in the proofs of the main results. Our approach is based on the method of spectral mappings (see [14]). Since a part of the proofs repeat the standard technique of [8, Sect. 1.6] and [13], we omit the details and focus on the differences of our methods from the classical ones.

Let us consider two BVPs $L=L(q(x), h, H)$ and $\tilde{L}=(\tilde{q}(x), \tilde{h}, \tilde{H})$ with different coefficients. Fix $N=N(L)$ and the contour $\gamma_{N}$. Assume that the eigenvalues $\left\{\tilde{\lambda}_{n}\right\}_{n=0}^{N}$ lie inside $\gamma_{N}$ and $\left\{\tilde{\lambda}_{n}\right\}_{n=N+1}^{\infty}$ lie outside $\gamma_{N}$.

Define

$$
D(x, \lambda, \xi):=\frac{\varphi(x, \lambda) \varphi^{\prime}(x, \xi)-\varphi^{\prime}(x, \lambda) \varphi(x, \xi)}{\lambda-\xi}=\int_{0}^{x} \varphi(t, \lambda) \varphi(t, \xi) d t .
$$


For $K \in \mathbb{N}$, consider the region $\Upsilon_{K}:=\left\{\lambda \in \mathbb{C}:-p<\operatorname{Re} \lambda<\left(K+\frac{1}{2}\right)^{2},|\operatorname{Im} \lambda|<p\right\}$ and its boundary $v_{K}:=\partial \Upsilon_{K}$ with the counter-clockwise circuit. The constant $p$ is chosen so that $\operatorname{Re} \lambda_{n}>-p, \operatorname{Re} \tilde{\lambda}_{n}>-p,\left|\operatorname{Im} \lambda_{n}\right|<p,\left|\operatorname{Im} \tilde{\lambda}_{n}\right|<p$ for all $n \geq 0$. Using the contour integration (see [8, p. 53] for details), we obtain the relation

$$
\varphi(x, \lambda)=\tilde{\varphi}(x, \lambda)+\lim _{K \rightarrow \infty} \frac{1}{2 \pi i} \oint_{v_{K}} D(x, \lambda, \xi) \hat{M}(\xi) \tilde{\varphi}(x, \xi) d \xi,
$$

where $\hat{M}:=\tilde{M}-M$. Applying the residue theorem and observing that the function $(\hat{M}(\lambda)-$ $\left.\hat{\mathcal{M}}_{N}(\lambda)\right)$ is analytic inside $\gamma_{N}$, we obtain the relation

$$
\begin{aligned}
\varphi(x, \lambda)= & \tilde{\varphi}(x, \lambda)+\frac{1}{2 \pi i} \oint_{\gamma_{N}} D(x, \lambda, \xi) \hat{\mathcal{M}}_{N}(\xi) \tilde{\varphi}(x, \xi) d \xi \\
& +\sum_{n=N+1}^{\infty}\left(\tilde{M}_{n} D\left(x, \lambda, \tilde{\lambda}_{n}\right) \tilde{\varphi}\left(x, \tilde{\lambda}_{n}\right)-M_{n} D\left(x, \lambda, \lambda_{n}\right) \tilde{\varphi}\left(x, \lambda_{n}\right)\right) .
\end{aligned}
$$

We use relation (3.1) for deriving the main equation of Inverse problem 2.1 in a special Banach space. Denote by $B_{C}$ the Banach space of functions continuous on $\gamma_{N}$ with the norm

$$
\left\|f_{C}\right\|_{B_{C}}=\max _{\lambda \in \gamma_{N}}\left|f_{C}(\lambda)\right|, \quad f_{C} \in B_{C} .
$$

Denote by $B_{D}$ the Banach space of bounded infinite sequences $f_{D}=\left[f_{n}\right]_{n=1}^{\infty}$ with the norm

$$
\left\|f_{D}\right\|_{B_{D}}=\sup _{n \geq 1}\left|f_{n}\right|, \quad f_{D} \in B_{D}
$$

Define the Banach space

$$
B:=\left\{f=\left(f_{C}, f_{D}\right): f_{C} \in B_{C}, f_{D} \in B_{D}\right\}, \quad\|f\|_{B}:=\left\|f_{C}\right\|_{C}+\left\|f_{D}\right\|_{D} .
$$

Here and below the lower indices $C$ and $D$ mean a "continuous" and a "discrete" part, respectively.

For every $x \in[0, \pi]$, define the element $\psi(x)=\left(\psi_{C}(x), \psi_{D}(x)\right)$, where

$$
\begin{aligned}
& \psi_{C}(x, \lambda)=\varphi(x, \lambda), \quad \lambda \in \gamma_{N}, \quad \psi_{D}(x)=\left[\psi_{n}(x)\right]_{n=1}^{\infty}, \\
& \psi_{2 j-1}(x)=\varphi\left(x, \tilde{\lambda}_{N+j}\right), \quad \psi_{2 j}(x)=\chi_{N+j}\left(\varphi\left(x, \lambda_{N+j}\right)-\varphi\left(x, \tilde{\lambda}_{N+j}\right)\right), \quad j \geq 1, \\
& \chi_{n}:= \begin{cases}\xi_{n}^{-1}, & \text { if } \xi_{n} \neq 0, \\
0, & \text { if } \xi_{n}=0 .\end{cases}
\end{aligned}
$$

The element $\tilde{\psi}(x)$ is defined analogously by using $\tilde{\varphi}$ instead of $\varphi$.

For the solution $\varphi(x, \lambda)$, the following standard asymptotics is valid:

$$
\varphi(x, \lambda)=\cos \rho x+O\left(\rho^{-1} \exp (|\operatorname{Im} \rho| x)\right), \quad|\rho| \rightarrow \infty,
$$


where $\rho=\sqrt{\lambda}, \operatorname{Re} \rho \geq 0$. Using (1.4) and (3.2), we obtain the estimates

$$
\left|\varphi\left(x, \lambda_{n}\right)\right| \leq C,\left|\varphi\left(x, \lambda_{n}\right)-\varphi\left(x, \tilde{\lambda}_{n}\right)\right| \leq C \xi_{n}, \quad x \in[0, \pi], n \geq 0,
$$

where the constant $C$ does not depend on $x$ and $n$. Analogous relations are valid for $\tilde{\varphi}(x, \lambda)$. Consequently, for each fixed $x \in[0, \pi]$, we have $\psi(x) \in B$ and $\tilde{\psi}(x) \in B$.

For each fixed $x \in[0, \pi]$, we define the linear bounded operator $R(x): B \rightarrow B$ as follows:

$$
\begin{aligned}
& R(x)=\left(\begin{array}{ll}
R_{C C}(x) & R_{C D}(x) \\
R_{D C}(x) & R_{D D}(x)
\end{array}\right), \\
& R_{C C}(x): B_{C} \rightarrow B_{C}, \quad R_{C D}(x): B_{D} \rightarrow B_{C}, \\
& R_{D C}(x): B_{C} \rightarrow B_{D}, \quad R_{D D}(x): B_{D} \rightarrow B_{D}, \\
& R(x) f=\left(R_{C C}(x) f_{C}+R_{C D}(x) f_{D}, R_{D C}(x) f_{C}+R_{D D}(x) f_{D}\right), \quad f=\left(f_{C}, f_{D}\right) \in B, \\
& \left(R_{C C}(x) f_{C}\right)(\lambda)=\frac{1}{2 \pi i} \oint_{\gamma_{N}} D(x, \lambda, \xi) \hat{\mathcal{M}}_{N}(\xi) f_{C}(\xi) d \xi, \\
& \left(R_{C D}(x) f_{D}\right)(\lambda)=\sum_{k=1}^{\infty}\left(\left(\tilde{M}_{N+k} D\left(x, \lambda, \tilde{\lambda}_{N+k}\right)-M_{N+k} D\left(x, \lambda, \lambda_{N+k}\right)\right) f_{2 k-1}\right. \\
& \left.-\xi_{N+k} M_{N+k} D\left(x, \lambda, \lambda_{N+k}\right) f_{2 k}\right) \text {, } \\
& \left(R_{D C}(x) f_{C}\right)_{2 j-1}=\frac{1}{2 \pi i} \oint_{\gamma_{N}} D\left(x, \tilde{\lambda}_{N+j}, \xi\right) \hat{\mathcal{M}}_{N}(\xi) f_{C}(\xi) d \xi \text {, } \\
& \left(R_{D C}(x) f_{C}\right)_{2 j}=\frac{1}{2 \pi i} \oint_{\gamma_{N}}\left(D\left(x, \lambda_{N+j}, \xi\right)-D\left(x, \tilde{\lambda}_{N+j}, \xi\right)\right) \chi_{N+j} \hat{\mathcal{M}}_{N}(\xi) f_{C}(\xi) d \xi \text {, } \\
& \left(R_{D D}(x) f_{D}\right)_{2 j-1}=\sum_{k=1}^{\infty}\left(\left(\tilde{M}_{N+k} D\left(x, \tilde{\lambda}_{N+j}, \tilde{\lambda}_{N+k}\right)-M_{N+k} D\left(x, \tilde{\lambda}_{N+j}, \lambda_{N+k}\right)\right) f_{2 k-1}\right. \\
& \left.-\xi_{N+k} M_{N+k} D\left(x, \tilde{\lambda}_{N+j}, \lambda_{N+k}\right) f_{2 k}\right) \text {, } \\
& \left(R_{D D}(x) f_{D}\right)_{2 j}=\chi_{N+j} \sum_{k=1}^{\infty}\left(\left(\tilde{M}_{N+k}\left(D\left(x, \lambda_{N+j}, \tilde{\lambda}_{N+k}\right)-D\left(x, \tilde{\lambda}_{N+j}, \tilde{\lambda}_{N+k}\right)\right)\right.\right. \\
& \left.-M_{N+k}\left(D\left(x, \lambda_{N+j}, \lambda_{N+k}\right)-D\left(x, \tilde{\lambda}_{N+j}, \lambda_{N+k}\right)\right)\right) f_{2 k-1} \\
& \left.-\xi_{N+k} M_{N+k}\left(D\left(x, \lambda_{N+j}, \lambda_{N+k}\right)-D\left(x, \tilde{\lambda}_{N+j}, \lambda_{N+k}\right)\right) f_{2 k}\right) \text {, }
\end{aligned}
$$

where $\lambda \in \gamma_{N}, j \geq 1, f_{D}=\left[f_{k}\right]_{k=1}^{\infty}$.

Taking $\lambda \in \gamma_{N}, \lambda=\tilde{\lambda}_{n}$ and $\lambda=\lambda_{n}, n>N$, in (3.1), we obtain the so-called main equation in the Banach space $B$ :

$$
\psi(x)=(I+R(x)) \tilde{\psi}(x), \quad x \in[0, \pi] .
$$

Here, $I$ is the identity operator in $B$.

Now suppose that the problem $L$ and the data $\tilde{G}=\left\{\tilde{\lambda}_{n}, \tilde{M}_{n}\right\}_{n=0}^{\infty}$ satisfy the conditions of Theorem 2.2. We choose $\delta_{0}$ to be so small that the values $\left\{\tilde{\lambda}_{n}\right\}_{n=0}^{N}$ definitely lie inside $\gamma_{N}$ and the values $\left\{\tilde{\lambda}_{n}\right\}_{n>N}$ definitely lie outside $\gamma_{N}$. It is not known whether the data $\tilde{G}$ 
correspond to any problem $\tilde{L}$ or not. Let $\psi(x)$ and $R(x)$ be constructed by $L$ and $\tilde{G}$ via the formulas above. Then the following assertion holds.

Lemma 3.1 For each fixed $x \in[0, \pi]$, the following estimate is valid:

$$
\|R(x)\|_{B \rightarrow B} \leq C \delta
$$

where the constant $C$ does not depend on $x, \delta$ and on the choice of $\tilde{G}$ satisfying the conditions of Theorem 2.2.

Proof In order to prove (3.6), it is sufficient to obtain similar estimates for $\left\|R_{C C}(x)\right\|_{B_{C} \rightarrow B_{C}}$, $\left\|R_{C D}(x)\right\|_{B_{D} \rightarrow B_{C}},\left\|R_{D C}(x)\right\|_{B_{C} \rightarrow B_{D}}$, and $\left\|R_{D D}(x)\right\|_{B_{D} \rightarrow B_{D}}$. Using (2.2) and (3.3), we get

$$
\left\|R_{C C}(x)\right\|_{B_{C} \rightarrow B_{C}} \leq \frac{1}{2 \pi} \text { length }\left(\gamma_{N}\right) \cdot \max _{\lambda, \xi \in \gamma_{N}}|D(x, \lambda, \xi)| \cdot \max _{\xi \in \gamma_{N}}\left|\hat{\mathcal{M}}_{N}(\xi)\right| \leq C \delta
$$

The standard estimates (see [8, Lemma 1.6.2]) imply

$$
\left|D\left(x, \lambda, \tilde{\lambda}_{n}\right)-D\left(x, \lambda, \lambda_{n}\right)\right| \leq \frac{C \exp (|\operatorname{Im} \rho| x) \xi_{n}}{|\rho-n|+1}, \quad n \geq 0 .
$$

Combining the latter relation with (3.4), (2.3) and the obvious estimates

$$
\left|M_{n}\right| \leq C, \quad\left|\tilde{M}_{n}-M_{n}\right| \leq \xi_{n}, \quad n \geq 0,
$$

we get

$$
\begin{aligned}
\left\|R_{C D}(x)\right\|_{B_{D} \rightarrow B_{C}} \leq & \max _{\lambda \in \gamma_{N}} \sum_{k=1}^{\infty}\left(\left|\tilde{M}_{N+k}-M_{N+k}\right|\left|D\left(x, \lambda, \tilde{\lambda}_{N+k}\right)\right|\right. \\
& +\left|M_{N+k}\right|\left|D\left(x, \lambda, \tilde{\lambda}_{N+k}\right)-D\left(x, \lambda, \lambda_{N+k}\right)\right| \\
& \left.+\xi_{N+k}\left|M_{N+k}\right|\left|D\left(x, \lambda, \lambda_{N+k}\right)\right|\right) \\
\leq & C \sum_{n=N+1}^{\infty} \frac{\xi_{n}}{|\rho-n|+1} \leq C\left(\sum_{n=N+1}^{\infty}\left(n \xi_{n}\right)^{2}\right)^{1 / 2} \leq C \delta .
\end{aligned}
$$

One can similarly study the components $R_{D C}(x)$ and $R_{D D}(x)$ and finally arrive at the assertion of the lemma.

Corollary 3.2 There exists $\delta_{0}>0$ such that, for every $\delta \leq \delta_{0}$ and $x \in[0, \pi]$, the estimate $\|R(x)\|_{B \rightarrow B} \leq \frac{1}{2}$ holds. In this case, for each fixed $x \in[0, \pi]$, the operator $(I+R(x))$ has a bounded inverse, and the main equation (3.5) has a unique solution.

\section{Proofs}

The aim of this section is to prove Theorems 2.2 and 2.3. Using the solution of the main equation (3.5), we construct the values $\tilde{q}(x), \tilde{h}$, and $\tilde{H}$ being the solution of Inverse problem 2.1 for $\tilde{G}$. Furthermore, stability estimates (2.4) are proved. We just outline the general strategy, since the detailed proofs are analogous to [8, Sect. 1.6.2]. 
Let $L$ be a fixed BVP, and let $\delta_{0}$ satisfy the conditions of Corollary 3.2. Let $\tilde{G}=\left\{\tilde{\lambda}_{n}, \tilde{M}_{n}\right\}_{n=0}^{\infty}$ be arbitrary fixed data satisfying the conditions of Theorem 2.2. Then the main equation (3.5) constructed by $L$ and $\tilde{G}$ has a unique solution for each fixed $x \in[0, \pi]$. Denote this solution by $\tilde{\psi}(x)=\left(\tilde{\psi}_{C}(x), \tilde{\psi}_{D}(x)\right), \tilde{\psi}_{C}(x)=\tilde{\psi}_{C}(x, \lambda), \tilde{\psi}_{D}(x)=\left[\tilde{\psi}_{n}(x)\right]_{n=1}^{\infty}$.

Lemma 4.1 The functions $\tilde{\psi}_{C}(x, \lambda)$ for each fixed $\lambda \in \gamma_{N}$ and $\tilde{\psi}_{n}(x)$ for $n \geq 1$ are continuously differentiable with respect to $x \in[0, \pi]$. Moreover, the following estimates hold for $x \in[0, \pi], v=0,1$ :

$$
\begin{aligned}
& \left|\tilde{\psi}^{(v)}(x, \lambda)\right| \leq C, \quad\left|\tilde{\psi}_{C}^{(v)}(x, \lambda)-\psi_{C}^{(v)}(x, \lambda)\right| \leq C \delta, \quad \lambda \in \gamma_{N}, \\
& \left|\tilde{\psi}_{n}^{(v)}(x)\right| \leq C n^{v}, \quad\left|\tilde{\psi}_{n}(x)-\psi_{n}(x)\right| \leq C \delta \eta_{n}, \quad\left|\tilde{\psi}_{n}^{\prime}(x)-\psi_{n}^{\prime}(x)\right| \leq C \delta, \quad n \geq 1, \\
& \eta_{n}:=\left(\sum_{k=1}^{\infty} \frac{1}{k^{2}(|n-k|+1)^{2}}\right)^{1 / 2},
\end{aligned}
$$

where the constant $C$ depends only on $L$ and $\delta_{0}$.

Define the function

$$
\begin{aligned}
\tilde{\varphi}(x, \lambda):= & \varphi(x, \lambda)-\frac{1}{2 \pi i} \oint_{\gamma_{N}} D(x, \lambda, \xi) \hat{\mathcal{M}}_{N}(\xi) \tilde{\psi}_{C}(x, \xi) d \xi \\
& -\sum_{k=1}^{\infty}\left(\tilde{M}_{N+k} D\left(x, \lambda, \tilde{\lambda}_{N+k}\right) \tilde{\psi}_{2 k-1}(x)\right. \\
& \left.-M_{N+k} D\left(x, \lambda, \lambda_{N+k}\right)\left(\tilde{\psi}_{2 k-1}(x)+\xi_{N+k} \tilde{\psi}_{2 k}(x)\right)\right) .
\end{aligned}
$$

It is easy to check that

$$
\begin{aligned}
& \tilde{\varphi}(x, \lambda)=\tilde{\psi}_{C}(x, \lambda), \quad \lambda \in \gamma_{N}, \\
& \tilde{\varphi}\left(x, \tilde{\lambda}_{N+k}\right)=\tilde{\psi}_{2 k-1}(x), \quad \tilde{\varphi}\left(x, \lambda_{N+k}\right)=\tilde{\psi}_{2 k-1}(x)+\xi_{N+k} \tilde{\psi}_{2 k}(x), \quad k \geq 1 .
\end{aligned}
$$

Consequently, Lemma 4.1 implies

$$
\left.\begin{array}{l}
\left|\tilde{\varphi}^{(v)}(x, \lambda)\right| \leq C, \quad\left|\tilde{\varphi}^{(v)}(x, \lambda)-\varphi^{(v)}(x, \lambda)\right| \leq C \delta, \quad \lambda \in \gamma_{N}, \\
\left|\tilde{\varphi}^{(v)}\left(x, \lambda_{N+k}\right)\right| \leq C k^{\nu}, \quad\left|\tilde{\varphi}\left(x, \lambda_{N+k}\right)-\varphi\left(x, \lambda_{N+k}\right)\right| \leq C \delta \eta_{k}, \\
\left|\tilde{\varphi}^{\prime}\left(x, \lambda_{N+k}\right)-\varphi^{\prime}\left(x, \lambda_{N+k}\right)\right| \leq C \delta, \quad k \geq 1, v=0,1, x \in[0, \pi] .
\end{array}\right\}
$$

Similar estimates also hold for $\lambda_{N+k}$ replaced by $\tilde{\lambda}_{N+k}$.

Introduce the functions

$$
\begin{aligned}
\varepsilon_{0}(x):= & \frac{1}{2 \pi i} \oint_{\gamma_{N}} \hat{\mathcal{M}}_{N}(\xi) \varphi(x, \xi) \tilde{\varphi}(x, \xi) d \xi+\sum_{n=N+1}^{\infty}\left(\tilde{M}_{n} \varphi\left(x, \tilde{\lambda}_{n}\right) \tilde{\varphi}\left(x, \tilde{\lambda}_{n}\right)\right. \\
& \left.-M_{n} \varphi\left(x, \lambda_{n}\right) \tilde{\varphi}\left(x, \lambda_{n}\right)\right), \quad \varepsilon(x):=-2 \varepsilon_{0}^{\prime}(x) .
\end{aligned}
$$

Using (2.2), (2.3), (3.7), (4.1), and (4.2), we prove the following lemma. 
Lemma 4.2 The integral $\int_{\gamma_{N}}(\cdots) d \xi$ and the series $\sum_{n=N+1}^{\infty}(\cdots)$ in (4.2) converge absolutely and uniformly with respect to $x \in[0, \pi]$. The function $\varepsilon_{0}$ is absolutely continuous on $[0, \pi]$ and $\varepsilon \in L_{2}(0, \pi)$. In addition,

$$
\max _{x \in[0, \pi]}\left|\varepsilon_{0}(x)\right| \leq C \delta, \quad\|\varepsilon\|_{L_{2}(0, \pi)} \leq C \delta .
$$

Define

$$
\tilde{q}(x)=q(x)+\varepsilon(x), \quad \tilde{h}=h-\varepsilon_{0}(0), \quad \tilde{H}=H+\varepsilon_{0}(\pi) .
$$

Lemma 4.2 implies that $\tilde{q} \in L_{2}(0, \pi)$ and estimates (2.4) hold. Consider the BVP $\tilde{L}=$ $L(\tilde{q}(x), \tilde{h}, \tilde{H})$ and the function

$$
\tilde{M}(\lambda):=\sum_{n \in \tilde{S}} \sum_{\nu=0}^{\tilde{m}_{n}-1} \frac{\tilde{M}_{n+\nu}}{\left(\lambda-\tilde{\lambda}_{n}\right)^{\nu+1}} .
$$

Lemma 4.3 The function $\tilde{M}(\lambda)$ is the Weyl function of $\tilde{L}$.

Thus, Corollary 3.2 and Lemmas 4.1-4.3 together prove Theorem 2.2.

Proof of Theorem 2.3 We prove Theorem 2.3 by reduction to Theorem 2.2. For simplicity, suppose that the problem $L$ has the only multiple eigenvalue $\lambda_{0}$ of multiplicity $m:=m_{0}$. The general case is completely similar, but more complicated technically. In our special case, we have

$$
\hat{\mathcal{M}}_{N}(\lambda)=\sum_{j=0}^{m-1} \frac{\tilde{M}_{j}}{\lambda-\tilde{\lambda}_{j}}-\sum_{j=0}^{m-1} \frac{M_{j}}{\left(\lambda-\lambda_{0}\right)^{j+1}}
$$

Using the obvious relation

$$
\frac{1}{\lambda-\tilde{\lambda}_{j}}=\frac{1}{\lambda-\lambda_{0}}\left(1+\frac{\tilde{\lambda}_{j}-\lambda_{0}}{\lambda-\tilde{\lambda}_{j}}\right)
$$

we obtain

$$
\frac{1}{\lambda-\tilde{\lambda}_{j}}=\sum_{s=0}^{2(m-1)} \frac{\left(\tilde{\lambda}_{j}-\lambda_{0}\right)^{s}}{\left(\lambda-\lambda_{0}\right)^{s+1}}+\frac{\left(\tilde{\lambda}_{j}-\lambda_{0}\right)^{2 m-1}}{\left(\lambda-\lambda_{0}\right)^{2 m-1}\left(\lambda-\tilde{\lambda}_{j}\right)} .
$$

Substituting (4.4) into (4.3), we derive

$$
\begin{aligned}
\hat{\mathcal{M}}_{N}(\lambda)= & \sum_{s=0}^{m-1} \frac{1}{\left(\lambda-\lambda_{0}\right)^{s+1}}\left(\sum_{j=0}^{m-1}\left(\tilde{\lambda}_{j}-\lambda_{0}\right)^{s} \tilde{M}_{j}-M_{s}\right) \\
& +\sum_{s=m}^{2(m-1)} \frac{1}{\left(\lambda-\lambda_{0}\right)^{s+1}}\left(\sum_{j=0}^{m-1}\left(\tilde{\lambda}_{j}-\lambda_{0}\right)^{s} \tilde{M}_{j}\right)+\sum_{j=0}^{m-1} \frac{\left(\tilde{\lambda}_{j}-\lambda_{0}\right)^{2 m-1} \tilde{M}_{j}}{\left(\lambda-\lambda_{0}\right)^{2 m-1}\left(\lambda-\tilde{\lambda}_{j}\right)} .
\end{aligned}
$$


For an appropriate choice of $\delta_{0}$, we have $\left|\lambda-\tilde{\lambda}_{j}\right| \geq c_{0}, j=\overline{0, m-1},\left|\lambda-\lambda_{0}\right| \geq c_{0}$ for $\lambda \in \gamma_{N}$, where the constant $c_{0}>0$ depends only on $L$ and $\delta_{0}$. Therefore, taking estimates (2.6) into account, we conclude that

$$
\max _{\lambda \in \gamma_{N}}\left|\hat{\mathcal{M}}_{N}(\lambda)\right| \leq C \delta
$$

Using the latter relation together with the other conditions of Theorem 2.3, we apply Theorem 2.2 and arrive at the assertion of Theorem 2.3.

Example 4.4 Suppose that $m_{0}=2, m_{n}=1$ for $n \geq 2$, i.e., $N=1$. Let us construct a special family of data $\tilde{G}=\left\{\tilde{\lambda}_{n}, \tilde{M}_{n}\right\}$ "close" to $G$ in the sense of Theorem 2.3. For simplicity, put $\tilde{\lambda}_{n}:=\lambda_{n}, \tilde{M}_{n}:=M_{n}$ for $n \geq 2$, i.e., only the first double eigenvalue $\lambda_{0}$ can be perturbed. In this case, condition (2.3) holds automatically for any $\delta>0$ and conditions (2.6) take the form

$$
\begin{aligned}
& \left|\tilde{M}_{0}+\tilde{M}_{1}-M_{0}\right| \leq \delta \\
& \left|\tilde{M}_{0}\left(\tilde{\lambda}_{0}-\lambda_{0}\right)+\tilde{M}_{1}\left(\tilde{\lambda}_{1}-\lambda_{0}\right)-M_{1}\right| \leq \delta, \\
& \left|\tilde{M}_{0}\left(\tilde{\lambda}_{0}-\lambda_{0}\right)^{2}+\tilde{M}_{1}\left(\tilde{\lambda}_{1}-\lambda_{0}\right)^{2}\right| \leq \delta \\
& \left|\tilde{\lambda}_{j}-\lambda_{0}\right| \leq \sqrt{\delta}, \quad\left|\tilde{M}_{j}\right| \leq \frac{1}{\sqrt{\delta}}, \quad j=0,1 .
\end{aligned}
$$

Fix $\delta>0$ and put

$$
\begin{aligned}
& \tilde{M}_{0}:=\frac{a}{\sqrt{\delta}}+M_{0}, \quad \tilde{M}_{1}:=-\frac{a}{\sqrt{\delta}}, \\
& \tilde{\lambda}_{0}:=\lambda_{0}+\sqrt{\delta}, \quad \tilde{\lambda}_{1}:=\lambda_{0}-\sqrt{\delta}+c \delta, \\
& a:=\frac{M_{1}}{2}, \quad c:=\frac{M_{0}}{a} .
\end{aligned}
$$

One can easily check that

$$
\tilde{M}_{0}\left(\tilde{\lambda}_{0}-\lambda_{0}\right)^{v}+\tilde{M}_{1}\left(\tilde{\lambda}_{1}-\lambda_{0}\right)^{v}=M_{\nu}, \quad v=0,1,
$$

which implies (4.5) and (4.6). It can be also checked that

$$
\begin{aligned}
& \left|\tilde{M}_{0}\left(\tilde{\lambda}_{0}-\lambda_{0}\right)^{2}+\tilde{M}_{1}\left(\tilde{\lambda}_{1}-\lambda_{0}\right)^{2}\right| \leq C \delta, \quad\left|\tilde{\lambda}_{j}-\lambda_{0}\right| \leq C \sqrt{\delta} \\
& \left|\tilde{M}_{j}\right| \leq \frac{C}{\sqrt{\delta}}, \quad j=0,1
\end{aligned}
$$

for $\delta \in\left(0, \delta_{0}\right]$, where $\delta_{0}$ is sufficiently small and the constant $C$ depends only on $L$ and $\delta_{0}$. Despite the constant $C$ in (4.7), we can apply Theorem 2.3 and conclude that, for sufficiently small $\delta_{0}>0$ and $\delta \in\left(0, \delta_{0}\right]$, there exists the solution $(\tilde{q}, \tilde{h}, \tilde{H})$ of Inverse problem 2.1 for the data $\tilde{G}$ and estimates (2.4) hold.

An interesting feature of this example is that the eigenvalues $\tilde{\lambda}_{0}, \tilde{\lambda}_{1}$ are $\sqrt{\delta}$-close to $\lambda_{0}$ and the generalized weight numbers $\tilde{M}_{0}, \tilde{M}_{1}$ are sufficiently large for sufficiently small $\delta$. Nevertheless, the potential $\tilde{q}$ is $C \delta$-close to $q$ in $L_{2}$-norm. Analogous examples can be constructed for $m_{0}>2$ and eigenvalues $\left\{\tilde{\lambda}_{n}\right\}_{n=0}^{m_{0}-1}$, being $\delta^{1 / m_{0}}$-close to $\lambda_{0}$. 


\section{Case of Dirichlet boundary conditions}

In this section, we formulate the results similar to Theorems 2.2 and 2.3 for the case of the Dirichlet boundary conditions. Since the proofs for different types of boundary conditions are quite similar, we provide only formulations in this section.

Consider the boundary problem $L_{0}=L_{0}(q(x))$ for equation (1.1) with the complexvalued potential $q \in L_{2}(0, \pi)$ and the Dirichlet boundary conditions

$$
y(0)=y(\pi)=0 .
$$

Denote by $\left\{\lambda_{n}\right\}_{n=1}^{\infty}$ the eigenvalues of $L$ counted with their multiplicities and numbered so that $\left|\lambda_{n}\right| \leq\left|\lambda_{n+1}\right|, n \geq 1$. Equal eigenvalues are consecutive.

The eigenvalue multiplicities $\left\{m_{n}\right\}_{n=1}^{\infty}$ for the problem $L_{0}$ are introduced similarly to the case of Robin boundary conditions (1.2). Note that the asymptotic formula (1.4) is valid for $n \geq 1$, so we choose the minimal $N=N\left(L_{0}\right)$ such that $m_{n}=1$ for $n>N$ and $\left|\lambda_{N}\right|<\left|\lambda_{N+1}\right|$. Fix the contour $\gamma_{N}=\{\lambda \in \mathbb{C}:|\lambda|=r\}, r \in\left(\left|\lambda_{N}\right|,\left|\lambda_{N+1}\right|\right)$.

Let $\Phi(x, \lambda)$ be the solution of equation (1.1) under the conditions $\Phi(0, \lambda)=1, \Phi(\pi, \lambda)=0$. The Weyl function is defined as $M(\lambda):=\Phi^{\prime}(0, \lambda)$. Define

$$
\begin{aligned}
& M_{n+v}:=\operatorname{Res}_{\lambda=\lambda_{n}}\left(\lambda-\lambda_{n}\right)^{v} M(\lambda), \quad n \in S, v=\overline{0, m_{n}-1}, \\
& S:=\left\{n \in \mathbb{N}: n=1 \text { or } \lambda_{n} \neq \lambda_{n-1}\right\} .
\end{aligned}
$$

The following inverse problem is analogous to Inverse problem 2.1.

Inverse problem 5.1 Given the data $G:=\left\{\lambda_{n}, M_{n}\right\}_{n=1}^{\infty}$ for the problem $L_{0}(q(x))$, find the potential $q$.

Consider the data $\tilde{G}:=\left\{\tilde{\lambda}_{n}, \tilde{M}_{n}\right\}_{n=1}^{\infty}$. Define the function $\hat{\mathcal{M}}_{N}(\lambda)$ via formulas (2.1), using the data $\left\{\lambda_{n}, M_{n}\right\}_{n=1}^{N}$ and $\left\{\tilde{\lambda}_{n}, \tilde{M}_{n}\right\}_{n=1}^{N}$ defined in this section and

$$
S_{N}=S \cap\{1, \ldots, N\}, \quad \tilde{S}_{N}=S \cap\{1, \ldots, N\} .
$$

Put $\rho_{n}:=\sqrt{\lambda_{n}}, \arg \rho_{n} \in\left[-\frac{\pi}{2}, \frac{\pi}{2}\right)$, and $\xi_{n}:=\left|\rho_{n}-\tilde{\rho}_{n}\right|+n^{-2}\left|M_{n}-\tilde{M}_{n}\right|$ for $n \geq 1$.

Theorem 5.2 Let $L_{0}=L_{0}(q(x))$ be a fixed BVP in the form (1.1), (5.1), $N=N\left(L_{0}\right), \gamma_{N}=$ $\gamma_{N}\left(L_{0}\right)$. Then there exists $\delta_{0}>0$ (depending on $\left.L_{0}\right)$ such that, for any $\delta \in\left(0, \delta_{0}\right]$ and any complex numbers $\tilde{G}=\left\{\tilde{\lambda}_{n}, \tilde{M}_{n}\right\}_{n=1}^{\infty}$ satisfying conditions (2.2) and (2.3), there exists a complexvalued function $\tilde{q} \in L_{2}(0, \pi)$ being the solution of Inverse problem 5.1 for $\tilde{G}$. Moreover,

$$
\|q-\tilde{q}\|_{L_{2}(0, \pi)} \leq C \delta
$$

where the constant $C>0$ depends only on $L_{0}$ and $\delta_{0}$.

Theorem 5.3 Let $L_{0}=L_{0}(q(x))$ be a fixed BVP in the form (1.1), (5.1), $N=N\left(L_{0}\right)$. Then there exists $\delta_{0}>0$ (depending on $L_{0}$ ) such that, for any $\delta \in\left(0, \delta_{0}\right]$ and any complex numbers $\tilde{G}=\left\{\tilde{\lambda}_{n}, \tilde{M}_{n}\right\}_{n=1}^{\infty}$ satisfying conditions (2.3), (2.5) for $n, k \geq 1$ and (2.6), there exists a complex-valued function $\tilde{q} \in L_{2}(0, \pi)$ being the solution of Inverse problem 5.1 for $\tilde{G}$. Moreover, estimate (5.2) holds. 
Remark 5.4 The arguments of Sects. 3 and 4 imply that Theorems 2.2 and 5.2 are also valid if we change $\hat{\mathcal{M}}_{N}(\lambda)$ to $\hat{M}(\lambda)$ in (2.2).

\author{
Acknowledgements \\ The author is grateful to anonymous referees for their valuable comments. \\ Funding \\ This work was supported by Grant 20-31-70005 of the Russian Foundation for Basic Research.
}

Availability of data and materials

Not applicable.

Competing interests

The author declares that she has no competing interests.

Authors' contributions

NPB is the sole author of this paper. The author has read and approved the final manuscript.

\title{
Publisher's Note
}

Springer Nature remains neutral with regard to jurisdictional claims in published maps and institutional affiliations.

Received: 17 January 2020 Accepted: 2 July 2020 Published online: 11 July 2020

\section{References}

1. Papageorgiou, N.S., Radulescu, V.D., Repovs, D.D.: Positive solutions for the perturbations of the Robin eigenvalue problem plus an indefinite potential. Discrete Contin. Dyn. Syst. 37(5), 2589-2618 (2017)

2. Bai, Y., Wang, W., Li, K.: Solvability and coerciveness of multi-point Sturm-Liouville problems with abstract linear functionals. Bound. Value Probl. 2019, Paper No. 17 (2019)

3. Bondarenko, N.: Spectral analysis of the matrix Sturm-Liouville operator. Bound. Value Probl. 2019, Paper No. 178 (2019)

4. Hwang, G.: The elliptic sinh-Gordon equation in a semi-strip. Adv. Nonlinear Anal. 8(1), 533-544 (2019)

5. Marchenko, V.A.: Sturm-Liouville Operators and Their Applications. Naukova Dumka, Kiev (1977) (Russian); English transl.: Birkhäuser (1986)

6. Levitan, B.M.: Inverse Sturm-Liouville Problems. Nauka, Moscow (1984) (Russian); English transl.: VNU Sci. Press, Utrecht (1987)

7. Pöschel, J., Trubowitz, E.: Inverse Spectral Theory. Academic Press, New York (1987)

8. Freiling, G., Yurko, V.: Inverse Sturm-Liouville Problems and Their Applications. Nova Science Publishers, Huntington (2001)

9. Gala, S., Liu, Q., Ragusa, M.A.: A new regularity criterion for the nematic liquid crystal flows. Appl. Anal. 91(9), $1741-1747$ (2012)

10. Gala, S., Ragusa, M.A.: Logarithmically improved regularity criterion for the Boussinesq equations in Besov spaces with negative indices. Appl. Anal. 95(6), 1271-1279 (2016)

11. Guo, Y., Wei, G., Yao, R.: An inverse three spectra problem for Sturm-Liouville operators. Bound. Value Probl. 2018, Paper No. 68 (2018)

12. Tkachenko, V:: Non-selfadjoint Sturm-Liouville operators with multiple spectra. In: Interpolation Theory, Systems Theory and Related Topics. Oper. Theory Adv. Appl., vol. 134, pp. 403-414. Birkhäuser, Basel (2002)

13. Buterin, S.A.: On inverse spectral problem for non-selfadjoint Sturm-Liouville operator on a finite interval. J. Math. Anal. Appl. 335(1), 739-749 (2007)

14. Yurko, V.A.: Method of Spectral Mappings in the Inverse Problem Theory. Inverse and III-Posed Problems Series. VNU Science, Utrecht (2002)

15. Buterin, S.A., Shieh, C.-T., Yurko, V.A.: Inverse spectral problems for non-selfadjoint second-order differential operators with Dirichlet boundary conditions. Bound. Value Probl. 2013, Paper No. 180 (2013)

16. Albeverio, S., Hryniv, R., Mykytyuk, Y.: On spectra of non-self-adjoint Sturm-Liouville operators. Sel. Math. New Ser. 13, 571-599 (2008)

17. Marletta, M., Weikard, R.: Weak stability for an inverse Sturm-Liouville problem with finite spectral data and complex potential. Inverse Probl. 21, 1275-1290 (2005)

18. Horvath, M., Kiss, M.: Stability of direct and inverse eigenvalue problems: the case of complex potentials. Inverse Probl. 27, 095007 (2011)

19. Bondarenko, N., Buterin, S.: On a local solvability and stability of the inverse transmission eigenvalue problem. Inverse Probl. 33, 115010 (2017)

20. Buterin, S., Kuznetsova, M.: On Borg's method for non-selfadjoint Sturm-Liouville operators. Anal. Math. Phys. 9 2133-2150 (2019)

21. Bondarenko, N.P.: Inverse Sturm-Liouville problem with analytical functions in the boundary condition. Open Math. 8(1), 512-528 (2020). https://doi.org/10.1515/math-2020-0188

22. Bondarenko, N.P.: Solvability and stability of the inverse Sturm-Liouville problem with analytical functions in the boundary condition. Math. Methods Appl. Sci. (2020). https://doi.org/10.1002/mma.6451 